\title{
Learning of Finite Unions of Tree Patterns with Internal Structured Variables from Queries
}

\author{
Satoshi MATSUMOTO $^{\dagger a)}$, Takayoshi SHOUDAI ${ }^{\dagger \dagger}$, Tomoyuki UCHIDA ${ }^{\dagger \dagger \dagger}$, \\ Tetsuhiro MIYAHARA ${ }^{\dagger \dagger}$, Members, and Yusuke SUZUKI ${ }^{\dagger \dagger \dagger}$, Nonmember
}

\begin{abstract}
SUMMARY A linear term tree is defined as an edge-labeled rooted tree pattern with ordered children and internal structured variables whose labels are mutually distinct. A variable can be replaced with arbitrary edgelabeled rooted ordered trees. We consider the polynomial time learnability of finite unions of linear term trees in the exact learning model formalized by Angluin. The language $L(t)$ of a linear term tree $t$ is the set of all trees obtained from $t$ by substituting arbitrary edge-labeled rooted ordered trees for all variables in $t$. Moreover, for a finite set $S$ of linear term trees, we define $L(S)=\bigcup_{t \in S} L(t)$. A target of learning, denoted by $\mathcal{T}_{*}$, is a finite set of linear term trees, where the number of edge labels is infinite. In this paper, for any set $\mathcal{T}_{*}$ of $m$ linear term trees $(m \geq 0)$, we present a query learning algorithm which exactly identifies $\mathcal{T}_{*}$ in polynomial time using at most $2 m n^{2}$ Restricted Subset queries and at most $m+1$ Equivalence queries, where $n$ is the maximum size of counterexamples. Finally, we note that finite sets of linear term trees are not learnable in polynomial time using Restricted Equivalence, Membership and Subset queries.

key words: exact learning, computational learning theory, finite union of tree pattern languages
\end{abstract}

\section{Introduction}

Large number of documents such as HTML/XML files are accessible on Internet. Since such documents have tree structures but no rigid structure, they are called treestructured data, which are represented by rooted trees with ordered children and edge labels. As an example of representations of tree-structured data, we give a sample document SAMPLE_Doc in. Fig. 1 and the rooted tree $T$ as a representation of SAMPLE_Doc in Fig. 2. In the fields of Web mining and text mining, many researchers have been developing data mining techniques based on machine learning methods for analyzing tree-structured data. If we can construct oracles which answer any query in practical time, we can design efficient and effective data mining tools based on query learning algorithms using such oracles. Therefore, from the viewpoint of computational and algorithmic learning theory, in this paper, we consider a polynomial time learnability of finite unions of ordered tree patterns with internal structured variables in the exact learning model formalized by Angluin [4].

Manuscript received March 30, 2007.

Manuscript revised June 30, 2007.

${ }^{\dagger}$ The author is with the Department of Mathematical Sciences, Tokai University, Hiratsuka-shi, 259-1292 Japan.

${ }^{\dagger \dagger}$ The author is with the Department of Informatics, Kyushu University, Fukuoka-shi, 819-0395 Japan.

${ }^{+\dagger \dagger}$ The authors are with the Graduate School of Information Sciences, Hiroshima City University, Hiroshima-shi, 731-3194 Japan.

a)E-mail: matumoto@ss.u-tokai.ac.jp

DOI: $10.1093 /$ ietisy/e91-d.2.222

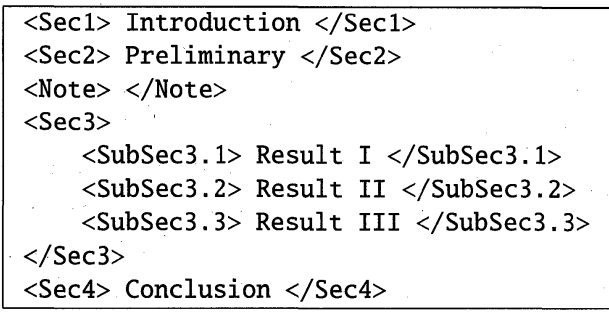

Fig. 1 A sample XML file SAMPLE_Doc.

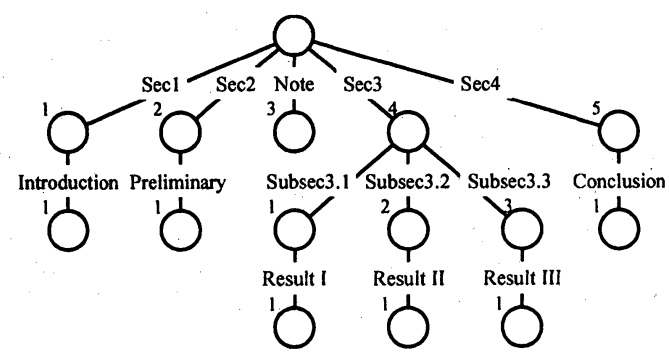

Fig. 2 A rooted ordered tree $T$.

As a knowledge representation for tree-structured data, we use a rooted ordered tree pattern with internal structured variables, called a term tree. A variable in a term tree is a pair of vertices for which an arbitrary rooted ordered tree can be substituted. As an example of term trees, we give a term tree $t$ in Fig. 3. By replacing the variables labeled with $x_{1}, x_{2}, x_{3}$ and $x_{4}$ of $t$ in Fig. 3 by ordered rooted trees $T_{1}, T_{2}, T_{3}$ and $T_{4}$, respectively, the rooted ordered tree $T$ in Fig. 2 can be obtained from $t, T_{1}, T_{2}, T_{3}$ and $T_{4}$. After Fig. 3, we assume that all children are ordered from left to right. Term trees are more powerful than or incomparable to other representations of tree-structured data, which were proposed in computational learning theory, such as ordered tree patterns [1] and ordered gapped tree patterns [6]. For example, in Fig. 3, the tree pattern $f(b, x, g(a, z), y)$ can be represented by the term tree $s$, but the term tree $t$ cannot be represented by any standard tree pattern because of the existence of internal structured variables represented by $x_{2}$ and $x_{3}$ in $t$

Let $\Lambda$ be an infinite set of edge labels. A term tree $t$ is said to be linear if all variable labels in $t$ are mutually distinct. In this paper, we deal with linear term trees only. By $\mu O \mathcal{T T}_{\Lambda}$ we denote the set of all linear term trees all of whose edge labels are in $\Lambda$. For a linear term tree 

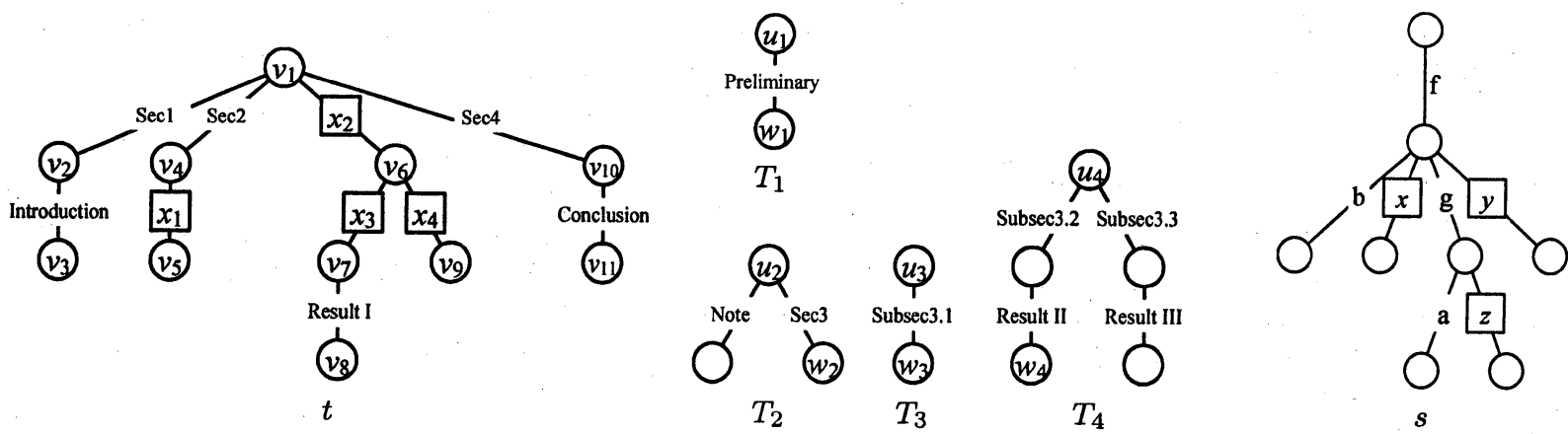

Fig. 3 Term trees $t, s$ and rooted ordered trees $T_{1}, T_{2}, T_{3}, T_{4}$. A variable is represented by a box with lines to its elements. The label inside a box is the variable label.

$t \in \mu O \mathcal{T T}_{\Lambda}$, the term tree language $L_{\Lambda}(t)$ of $t$ is the set of all rooted ordered trees which are obtained from $t$ by substituting arbitrary rooted ordered trees for all variables in $t$. We remark that the subtrees which are obtained from $t$ by removing the variables in $t$ represent common subtree structures in the trees in $L_{\Lambda}(t)$. In order to give a learning algorithm for tree-structured data including multiple or heterogeneous sources, we deal with finite unions of term tree languages of linear term trees in $\mu O \mathcal{T T}_{\Lambda}$. The language represented by a finite set of $k$ linear term trees $H=\left\{t_{1}, t_{2}, \ldots, t_{k}\right\}$ is the union of $k$ term tree languages of $t_{1}, t_{2}, \ldots, t_{k}$, i.e., $L_{\Lambda}(H)=L_{\Lambda}\left(t_{1}\right) \cup L_{\Lambda}\left(t_{2}\right) \cup \ldots \cup L_{\Lambda}\left(t_{k}\right)$. The class $\mu O \mathcal{T F} \mathcal{F}_{\Lambda}$ is defined as the collection of all finite sets of linear term trees in $\mu \mathcal{O T T}_{\Lambda}$. Our object in this paper is to make clear the polynomial time learnability of the class $\mu O \mathcal{T F} \mathcal{F}_{\Lambda}$ in exact learning model.

In exact learning model using queries, a learning algorithm accesses to oracles, which answer specific kinds of queries, and collects information about an unknown target. A learning algorithm is said to exactly learn a target set of linear term trees $\mathcal{T}_{*}$ in polynomial time if it outputs a set $S$ of linear term trees in $\mu O \mathcal{T T}_{\Lambda}$ in polynomial time such that $L_{\Lambda}(S)=L_{\Lambda}\left(\mathcal{T}_{*}\right)$, after it uses some queries and additional information. Since an edge label in tree structured data such as HTML/XML files is a word, we assume that the set of edge labels attached to edges of input trees is infinite. When a finite set $S$ of linear term trees in $\mu O \mathcal{T T}_{\Lambda}$ is given as an input, an oracle for Restricted Subset queries answers "yes" if $L_{\Lambda}(S) \subseteq L_{\Lambda}\left(\mathcal{T}_{*}\right)$, and answers "no", otherwise. An oracle for Equivalence queries returns "yes" if $L_{\Lambda}(S)=L_{\Lambda}\left(\mathcal{T}_{*}\right)$, and returns a counterexample in $\left(L_{\Lambda}(S) \cup L_{\Lambda}\left(\mathcal{T}_{*}\right)\right)-\left(L_{\Lambda}(S) \cap L_{\Lambda}\left(\mathcal{T}_{*}\right)\right)$, otherwise.

In this paper, we show that any finite union of languages defined by $m$ linear term trees is exactly identifiable in polynomial time using at most $2 m n^{2}$ Restricted Subset queries and at most $m+1$ Equivalence queries, where $n$ is the maximum size of counterexamples and the set of edge labels is infinite. The idea of our learning algorithm is as follows: First, we get a counterexample by an Equivalence query. Second, we generalize the counterexample by trying to replace an edge with a variable repeatedly as much as possible, in order to determine the number of edges in one of the linear term trees in the target set. Third, by trying to replace two adjacent variables with one variable repeatedly as much as possible, we determine the number of variables in the target linear term tree. We repeat these three steps until all linear term trees in the target set are found.

An oracle for Restricted Equivalence queries returns "yes" for an input set $S$ if $L_{\Lambda}(S)=L_{\Lambda}\left(\mathcal{T}_{*}\right)$, and answers "no", otherwise. An oracle for Membership queries answers "yes" for a rooted ordered tree $T$ if $T \in L_{\Lambda}\left(\mathcal{T}_{*}\right)$, and answers "no", otherwise. An oracle for Subset queries answers "yes" for an input set $S$ if $L_{\Lambda}(S) \subseteq L_{\Lambda}\left(\mathcal{T}_{*}\right)$, and returns a counterexample in $L_{\Lambda}(S)-L_{\Lambda}\left(\mathcal{T}_{*}\right)$, otherwise. Then, we show that finite sets of linear term trees are not learnable in polynomial time using Restricted Equivalence, Membership and Subset queries.

As previous work [10], we showed that the class of languages defined by restricted string patterns are exactly learnable using Membership queries and a positive example. In [11], we showed that unions of string subsequences are exactly learnable using Membership and Equivalence queries. Also our work [8], [15], [17] gave some classes of languages defined by extended term trees which are polynomial time inductively inferable from positive data. As an application [14], we proposed a data mining method from tree-structured data, using a learning algorithm for linear term trees based on another learning model [3]. As other related works, the works [1], [2], [5], [6] show the learnability of tree-structured pattern in exact learning model using queries. Especially, Arimura et al. showed in [5] that the unions of languages defined by ordered tree patterns are exactly learnable in polynomial time using Equivalence and Membership queries. Moreover, Arimura et al. showed in [6] that the unions of languages defined by $m$ ordered gapped tree patterns are exactly learnable in polynomial time using $O(m)$ Equivalence queries and $O\left(m n^{2}\right)$ Restricted Subset queries, where $n$ is the maximum size of counterexamples.

This paper is organized as follows. In Sect. 2, we introduce a linear term tree as a tree-structured pattern and a term tree language. Next, we briefly explain an exact learning model using queries. In Sect. 3, we show that any finite union of languages defined by linear term trees is exactly identifiable in polynomial time using Restricted Subset queries and Equivalence queries. In Sect. 4, we show fi- 
nite sets of linear term trees are not learnable in polynomial time using Restricted Equivalence, Membership and Subset queries.

\section{Preliminaries}

In this section, we define a term tree which is a treestructured pattern and then define a term tree language of a term tree. Next, we introduce an exact learning model using queries due to Angluin [4].

For a set $S$, the number of elements in $S$, called the size of $S$, is denoted by $|S|$.

\subsection{Term Trees and Term Tree Languages}

Let $X$ be an infinite alphabet whose element is called a variable label, and $\Lambda$ an alphabet where $\Lambda \cap X=\emptyset$. We call an element in $\Lambda$ an edge label, and in this paper, we assume that $|\Lambda|$ is infinite.

Definition 1: Let $T=\left(V_{T}, E_{T}\right)$ be an edge-labeled rooted tree which has a set $V_{T}$ of vertices and a set $E_{T}$ of edges labeled with elements of $\Lambda \cup X$. Let $H_{t}$ be the set of all edges in $E_{T}$ whose labels are in $X$. Let $V_{t}=V_{T}$ and $E_{t}=E_{T}-H_{t}$ (i.e., $E_{t} \cup H_{t}=E_{T}$ and $E_{t} \cap H_{t}=\emptyset$ ). A triplet $t=\left(V_{t}, E_{t}, H_{t}\right)$ is called a term tree, and elements in $V_{t}, E_{t}$ and $H_{t}$ are called a vertex, an edge and a variable, respectively.

For a term tree $t=\left(V_{t}, E_{t}, H_{t}\right)$ and its vertices $v_{1}$ and $v_{i}$, a path from $v_{1}$ to $v_{i}$ is a sequence $v_{1}, v_{2}, \ldots, v_{i}$ of distinct vertices of $t$ such that for any $j$ with $1 \leq j<i$, there exists an edge or a variable which consists of $v_{j}$ and $v_{j+1}$. If there is an edge which consists of $v$ and $v^{\prime}$ such that $v$ lies on the path from the root to $v^{\prime}$, then $v$ is said to be the parent of $v^{\prime}$ and $v^{\prime}$ is a child of $v$. We denote by $\left(v, v^{\prime}\right)$ the edge in $E_{t}$. If there is a variable which consists of $v$ and $v^{\prime}$ such that $v$ lies on the path from the root to $v^{\prime}$, Then $v$ is said to be the parent port of $v^{\prime}$ and $v^{\prime}$ is a child port of $v$. We denote by $\left[v, v^{\prime}\right]$ the variable in $H_{t}$.

A term tree $t$ is called ordered if every internal vertex $u$ in $t$ has a total ordering on all children of $u$. We define the size of $t$ as the number of vertices in $t$ and denote it by $|t|$, that is, $|t|=\left|V_{t}\right|$. For example, the ordered term tree $t=$ $\left(V_{t}, E_{t}, H_{t}\right)$ in Fig. 3 is defined as follows. $V_{t}=\left\{v_{1}, \ldots, v_{11}\right\}$, $E_{t}=\left\{\left(v_{1}, v_{2}\right),\left(v_{2}, v_{3}\right),\left(v_{1}, v_{4}\right),\left(v_{7}, v_{8}\right),\left(v_{1}, v_{10}\right),\left(v_{10}, v_{11}\right)\right\}$ with the root $v_{1}$ and the sibling relation displayed in Fig. 3 . $H_{t}=\left\{\left[v_{4}, v_{5}\right],\left[v_{1}, v_{6}\right],\left[v_{6}, v_{7}\right],\left[v_{6}, v_{9}\right]\right\}$.

We call an ordered term tree a term tree simply. In particular, a term tree $t=\left(V_{t}, E_{t}, H_{t}\right)$ is said to be linear if all variables in $H_{t}$ have mutually distinct variable labels in $X$. A linear term tree in this paper is a special form of a linear term tree of the previous work [18]. A variable in a linear term tree is also called a read-once variable and a formula with read-once variables is called a $\mu$-formula in computational learning theory. In this paper, we deal with linear term trees only. We denote by $\mu O \mathcal{T T}_{\Lambda}$ the set of all linear term trees with $\Lambda$ as a set of edge labels, and by $\mu O \mathcal{T F}_{\Lambda}$ the set of all finite sets of linear term trees with $\Lambda$ as a set of edge labels, that is, $\mu O \mathcal{T F}_{\Lambda}=\left\{S \subseteq \mu O \mathcal{T T}_{\Lambda}|| S \mid\right.$ is finite. $\}$. A linear term tree with no variable is called a ground term tree and considered to be a tree with ordered children. $O \mathcal{T}_{\Lambda}$ denotes the set of all ground term trees with $\Lambda$ as a set of edge labels.

For any linear term tree $t$, a vertex $u$ of $t$, and two children $u^{\prime}$ and $u^{\prime \prime}$ of $u$, we write $u^{\prime}<_{u}^{t} u^{\prime \prime}$ if $u^{\prime}$ is smaller than $u^{\prime \prime}$ in the order of the children of $u$. Let $f=\left(V_{f}, E_{f}, H_{f}\right)$ and $g=\left(V_{g}, E_{g}, H_{g}\right)$ be linear term trees. We say that $f$ and $g$ are isomorphic, denoted by $f \equiv g$, if there is a bijection $\varphi$ from $V_{f}$ to $V_{g}$ such that (i) the root of $f$ is mapped to the root of $g$ by $\varphi$, (ii) $\left(u, u^{\prime}\right) \in E_{f}$ if and only if $\left(\varphi(u), \varphi\left(u^{\prime}\right)\right) \in E_{g}$ and the two edges have the same edge label, (iii) $\left[u, u^{\prime}\right] \in H_{f}$ if and only if $\left[\varphi(u), \varphi\left(u^{\prime}\right)\right] \in H_{g}$, and (iv) for any vertex $u$ in $f$ which has more than one child, and for any two children $u^{\prime}$ and $u^{\prime \prime}$ of $u, u^{\prime}<_{u}^{f} u^{\prime \prime}$ if and only if $\varphi\left(u^{\prime}\right)<_{\varphi(u)}^{g} \varphi\left(u^{\prime \prime}\right)$. Two isomorphic linear term trees are considered to be identical.

Let $f$ and $g$ be linear term trees with at least two vertices. Let $h=\left[v, v^{\prime}\right]$ be a variable in $f$ with a variable label $x$ and $\sigma=\left[u, u^{\prime}\right]$ a list of two distinct vertices in $g$, where $u$ is the root of $g$ and $u^{\prime}$ is a leaf of $g$. The form $x:=[g, \sigma]$ is called a binding for $x$. A new linear term tree $f^{\prime}=f\{x:=[g, \sigma]\}$ is obtained by applying the binding $x:=[g, \sigma]$ to $f$ in the following way. Let $g^{\prime}$ be a copy of $g$ and $w, w^{\prime}$ the vertices of $g^{\prime}$ corresponding to $u, u^{\prime}$ of $g$, respectively. For the variable $h=\left[v, v^{\prime}\right]$, we attach $g^{\prime}$ to $f$ by removing the variable $h$ from $H_{f}$ and by identifying the vertices $v, v^{\prime}$ with the vertices $w, w^{\prime}$ of $g^{\prime}$ respectively. All variable in $f^{\prime}$ are labeled by mutually distinct variable labels in $X$.

A substitution $\theta$ is a finite collection of bindings $\left\{x_{1}:=\right.$ $\left.\left[g_{1}, \sigma_{1}\right], \cdots, x_{n}:=\left[g_{n}, \sigma_{n}\right]\right\}$, where $x_{i}$ 's are mutually distinct variable labels in $X$. The linear term tree $f \theta$, called the instance of $f$ by $\theta$, is obtained by applying all the bindings $x_{i}:=\left[g_{i}, \sigma_{i}\right]$ on $f$ simultaneously. We define a new total ordering $<_{v}^{f \theta}$ on every vertex $v$ in $f \theta$ in the following natural way. Suppose that $v$ has more than one child and let $v^{\prime}$ and $v^{\prime \prime}$ be two children of $v$ in $f \theta$. There are five cases in which the ordering between $v^{\prime}$ and $v^{\prime \prime}$ have to be newly defined. (1) $v \in V_{f \theta}-V_{f}$ : In this case, there is a linear term tree $g \in\left\{g_{1}, \cdots, g_{n}\right\}$ such that all of $v, v^{\prime}, v^{\prime \prime}$ are in $V_{g}$. Then $v^{\prime}<_{v}^{f \theta} v^{\prime \prime}$ is defined if and only if $v^{\prime}<_{v}^{g} v^{\prime \prime}$. If $v \in V_{f}$, we have the following four subcases. (2) $v^{\prime} \in V_{f}$ and $v^{\prime \prime} \in V_{f}$ : $v^{\prime}<_{v}^{f \theta} v^{\prime \prime}$ is defined if and only if $v^{\prime}<_{v}^{f} v^{\prime \prime}$. (3) $v^{\prime} \in V_{f}$ and there is a linear term tree $g \in\left\{g_{1}, \cdots, g_{n}\right\}$ such that $v^{\prime \prime} \in V_{g}$ : Let $w$ be the child port of the variable for which $g$ is substituted. We note that $v$ is the parent port of the variable. Then $v^{\prime}<_{v}^{f \theta} v^{\prime \prime}$ is defined if and only if $v^{\prime}<_{v}^{f} w$. (4) There is a linear term tree $g \in\left\{g_{1}, \cdots, g_{n}\right\}$ such that both of $v^{\prime}$ and $v^{\prime \prime}$ are in $V_{g}$ : Since $v$ is identified with the root of $g$ (say $u$ ), $v^{\prime}<_{v}^{f \theta} v^{\prime \prime}$ is defined if and only if $v^{\prime}<_{u}^{g} v^{\prime \prime}$. (5) There are two distinct linear term trees $g, g^{\prime} \in\left\{g_{1}, \cdots, g_{n}\right\}$ such that $v^{\prime} \in V_{g}$ and $v^{\prime \prime} \in V_{g^{\prime}}$ : Let $w$ (resp. $w^{\prime}$ ) be the child port of the variable for which $g$ (resp. $g^{\prime}$ ) is substituted. Then $v^{\prime}<_{v}^{f \theta} v^{\prime \prime}$ is defined if and only if $w<_{v}^{f} w^{\prime \prime}$. In Fig. 4, we give an example of the new ordering on vertices in a linear term tree. 

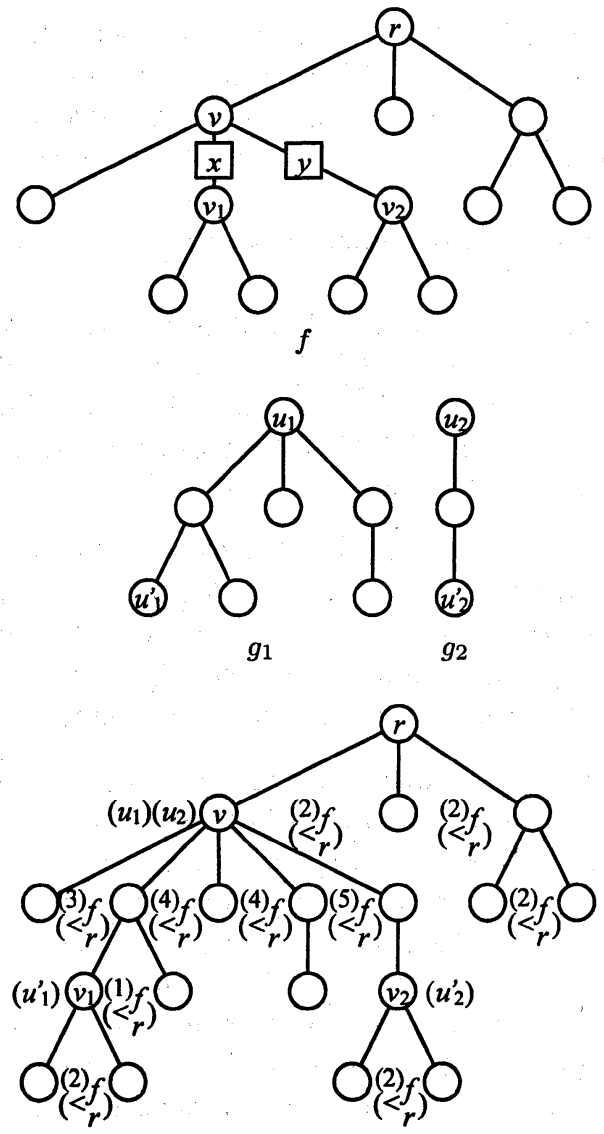

$$
f^{\prime}=f\left\{x:=\left[g_{1},\left[u_{1}, u_{1}^{\prime}\right]\right], y:=\left[g_{2},\left[u_{2}, u_{2}^{\prime}\right]\right]\right\}
$$

Fig. 4 The new ordering on vertices in the linear term tree $f^{\prime}=f\{x:=$ $\left.\left[g_{1},\left[u_{1}, u_{1}^{\prime}\right]\right], y:=\left[g_{2},\left[u_{2}, u_{2}^{\prime}\right]\right]\right\}$.

We define the root of the resulting linear term tree $f \theta$ as the root of $f$. Consider the examples in Fig. 2 and 3. An example of a linear term tree $t$ is given. Let $\theta=\left\{x_{1}:=\right.$ $\left[T_{1},\left[u_{1}, w_{1}\right]\right], x_{2}:=\left[T_{2},\left[u_{2}, w_{2}\right]\right], x_{3}:=\left[T_{3},\left[u_{3}, w_{3}\right]\right], x_{4}:=$ $\left.\left[T_{4},\left[u_{4}, w_{4}\right]\right]\right\}$ be a substitution, where $T_{1}, T_{2}, T_{3}$, and $T_{4}$ are ground term trees in Fig. 3. Then the instance $t \theta$ of the linear term tree $t$ by $\theta$ is isomorphic to the tree $T$ in Fig. 2. Let $f$ and $g$ be linear term trees. We write $f \leq g$ if there exists a substitution $\theta$ such that $f \equiv g \theta$. If $f \leq g$ and $f \neq \equiv$, then write $f<g$.

Definition 2: The term tree language $L_{\Lambda}(t)$ of a linear term tree $t \in \mu O \mathcal{T T}_{\Lambda}$ is $\left\{s \in O \mathcal{T}_{\Lambda} \mid s \leq t\right\}$. For a subset $S$ of $\mu O \mathcal{T T}_{\Lambda}$, we define $L_{\Lambda}(S)=\bigcup_{t \in S} L_{\Lambda}(t)$, and $L_{\Lambda}(S)$ is called the term tree language defined by $S$. In particular, we define $L_{\Lambda}(\emptyset)=\emptyset$.

Let $t$ be a linear term tree in $\mu O \mathcal{T T}_{\Lambda}$ and $S$ a set in $\mu O \mathcal{T F}_{\Lambda}$. Then we consider the following property: $L_{\Lambda}(t) \subseteq$ $L_{\Lambda}(h)$ for some $h \in S$ if and only if $L_{\Lambda}(t) \subseteq L_{\Lambda}(S)$. This property is important in the learning of unions of languages, is called compactness, which was proposed in [6], [7] and is enjoyed by similar languages, e.g., monotone DNF.

Lemma 1: Let $t$ be a linear term tree in $\mu O \mathcal{T T}_{\Lambda}, S$ a set in $\mu O \mathcal{T F}_{\Lambda}$ and $|\Lambda|$ infinite. Then, $t \leq h$ for some $h \in S$ if and

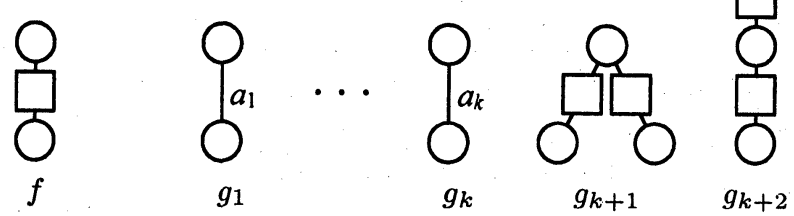

Fig. 5 An example which violates the property compactness: If $\Lambda=$ $\left\{a_{1}, \cdots, a_{k}\right\}(k \geq 1)$, then these linear term trees satisfy $L_{\Lambda}(f)=L_{\Lambda}\left(g_{1}\right) \cup$ $\cdots \cup L_{\Lambda}\left(g_{k+2}\right)$, but $L_{\Lambda}(f) \nsubseteq L_{\Lambda}\left(g_{i}\right)$ for all $i(1 \leq i \leq k+2)$.

only if $L_{\Lambda}(t) \subseteq L_{\Lambda}(S)$.

Proof. The only if part is obvious. Then, we only prove the if part. Since $|\Lambda|$ is infinite, there is an edge label $c$ which does not appear in any linear term tree in $S$. Let $w_{t}$ be a ground term tree obtained from $t$ by substituting all the variables in $t$ with edges labeled with $c$. Since $w_{t} \in L_{\Lambda}(t)$ and $L_{\Lambda}(t) \subseteq L_{\Lambda}(S)$, there exists a linear term tree $t^{\prime} \in S$ such that $w_{t} \in L_{\Lambda}\left(t^{\prime}\right)$. Since the edge label $c$ does not appear in $S$, we have $t \leq t^{\prime}$ by inverting the substitutions.

If $|\Lambda|$ is finite, then $\mu O \mathcal{T F} \mathcal{F}_{\Lambda}$ does not satisfies the property of compactness (see Fig. 5). By Lemma 1, $\mu O \mathcal{T F}_{\Lambda}$ satisfies the property of compactness if $|\Lambda|$ is infinite.

\subsection{Learning Model}

Let $\mathcal{T}_{*} \in \mu O \mathcal{T F}_{\Lambda}$ to be identified, and we say that the set $\mathcal{T}_{*}$ is a target. First we introduce the exact learning model via queries due to Angluin [4]. In this model, learning algorithms can access to oracles that will answer queries about the target set $L_{\Lambda}\left(\mathcal{T}_{*}\right)$. A learning algorithm collects information about $\mathcal{T}_{*}$ by using queries. We consider the following queries.

1. Membership query: The input is a ground term tree $T$ in $O \mathcal{T}_{\Lambda}$. The output is "yes" if $T \in L_{\Lambda}\left(\mathcal{T}_{*}\right)$, and "no" otherwise. The oracle which answers the Membership query is called a Membership oracle.

2. Subset query and Restricted Subset query: The input of both queries is a set $S$ in $\mu O \mathcal{T F}_{\Lambda}$. The output of Subset query is "yes" if $L_{\Lambda}(S) \subseteq L_{\Lambda}\left(\mathcal{T}_{*}\right)$, otherwise, a ground term tree, called a counterexample, in $L_{\Lambda}(S)$ $L_{\Lambda}\left(\mathcal{T}_{*}\right)$. The oracle which answers the Subset query is called a Subset oracle. The output of Restricted Subset query is "yes" if $L_{\Lambda}(S) \subseteq L_{\Lambda}\left(\mathcal{T}_{*}\right)$, otherwise "no". The oracle which answers the Restricted Subset query is called a Restricted Subset oracle.

3. Equivalence query and Restricted Equivalence query: The input of both queries is a set $S$ in $\mu O \mathcal{O T} \mathcal{F}_{\Lambda}$. The output of Equivalence query is "yes" if $L_{\Lambda}(S)=L_{\Lambda}\left(\mathcal{T}_{*}\right)$, otherwise a ground term tree, called a counterexample, in $\left(L_{\Lambda}(S) \cup L_{\Lambda}\left(\mathcal{T}_{*}\right)\right)-\left(L_{\Lambda}(S) \cap L_{\Lambda}\left(\mathcal{T}_{*}\right)\right)$. The oracle which answers the Equivalence query is called an Equivalence oracle. The output of Restricted Equivalence query is "yes" if $L_{\Lambda}(S)=L_{\Lambda}\left(\mathcal{T}_{*}\right)$, otherwise 
"no". The oracle which answers the Restricted Equivalence query is called a Restricted Equivalence oracle.

A learning algorithm $\mathcal{A}$ is said to exactly identify a target $\mathcal{T}_{*}$ in polynomial time if $\mathcal{A}$ outputs a set $S \in \mu O \mathcal{T F} \mathcal{F}_{\Lambda}$ in polynomial time such that $L_{\Lambda}(S)=L_{\Lambda}\left(\mathcal{T}_{*}\right)$.

For two linear term trees $t$ and $t^{\prime}$, we write $t \approx t^{\prime}$ if $\tilde{t} \equiv \tilde{t^{\prime}}$, where $\tilde{t}$ and $\tilde{t}^{\prime}$ are linear term trees obtained from $t$ and $t^{\prime}$ by replacing all edges with variables, respectively. For a set of linear term trees $H, N(H)$ denotes the total size of all linear term trees in $H$. The above oracles are implemented practically. We have the following propositions 1,2 and 3 with respect to the time complexities of Membership query, Restricted Subset query and Restricted Equivalence query, respectively.

Proposition 1 ([16]): Given a set of linear term trees $H$ and a ground term tree $T$, the problem of deciding whether or not $T \in L_{\Lambda}(H)$ is solvable in $O(N(H)|T|)$ time.

In order to show Proposition 2, we give the following lemma.

Lemma 2: Given two linear term trees $t$ and $t^{\prime}$, if $|\Lambda|$ is infinite then the problem of deciding whether or not $t \leq t^{\prime}$ is solvable in $O\left(|t|\left|t^{\prime}\right|\right)$ time.

Proof. Let $c$ be an edge label which does not appear in both $t$ and $t^{\prime}$. Here, we call an edge labeled with $c$ a $c$-edge simply. Let $T$ be the ground term tree obtained from $t$ by replacing all variables in $t$ with $c$-edges. We show the next claim: $T \in L_{\Lambda}\left(t^{\prime}\right)$ if and only if $t \preceq t^{\prime}$. The if direction is obvious. Then, we assume $T \in L_{\Lambda}\left(t^{\prime}\right)$. There exists a substitution $\theta$ satisfying $t^{\prime} \theta \equiv T$. Since no $c$-edge appear in $t^{\prime}$, all $c$-edges in $T$ must be $c$-edges of substituted trees in $\theta$. Let $\theta^{\prime}$ be the substitution obtained from $\theta$ by replacing all $c$-edges with variables. Then, since $t^{\prime} \theta^{\prime} \approx t^{\prime} \theta$ and $T \approx t$, it is easy to see that $t^{\prime} \theta^{\prime} \equiv t$. Therefore, we have $t \leq t^{\prime}$. Then, from this claim and Proposition 1, this lemma holds.

Proposition 2: Given two sets of linear term trees $H$ and $H^{\prime}$, if $|\Lambda|$ is infinite then the problem of deciding whether or not $L_{\Lambda}(H) \subseteq L_{\Lambda}\left(H^{\prime}\right)$ is solvable in $O\left(N(H) N\left(H^{\prime}\right)\right)$ time.

Proof. From Lemma $1, L_{\Lambda}(t) \subseteq L_{\Lambda}\left(H^{\prime}\right)$ if and only if there exists a linear term tree $t^{\prime} \in H^{\prime}$ with $t \leq t^{\prime}$. Then, from Lemma 2, the problem of deciding whether or not $L_{\Lambda}(t) \subseteq L_{\Lambda}\left(H^{\prime}\right)$ is solvable in $O\left(|t| N\left(H^{\prime}\right)\right)$ time. Therefore, the problem is solvable in $O\left(N(H) N\left(H^{\prime}\right)\right)$ time.

From Proposition 2 , it is easy to see that the following proposition holds.

Proposition 3: Given two sets of linear term trees $H$ and $H^{\prime}$, if $|\Lambda|$ is infinite then the problem of deciding whether or not $L_{\Lambda}(H)=L_{\Lambda}\left(H^{\prime}\right)$ is solvable in $O\left(N(H) N\left(H^{\prime}\right)\right)$ time.

If $|\Lambda|$ is infinite, then, for two sets of linear term trees $H$ : and $H^{\prime}$ such that $L_{\Lambda}(H) \nsubseteq L_{\Lambda}\left(H^{\prime}\right)$, there exists a linear term tree $t \in H$ such that $t \npreceq t^{\prime}$ for any $t^{\prime} \in H^{\prime}$. Let $c$ be an edge label which does not appear in any linear term tree in $H^{\prime}$. Let $T$ be the ground term tree which is obtained from $t$ by replacing all variables with edges of label $c$. It is easy to see that $T \in L_{\Lambda}(H)-L_{\Lambda}\left(H^{\prime}\right)$. From these observations, we can see that Subset query and Equivalence query have also square time complexities with respect to the input size.

\section{Learning Finite Unions of Term Tree Languages}

By presenting a learning algorithm LEARN_UNION in Fig. 6, we give the following theorem as one of the main results of this paper. The formal definitions of notations used in the algorithm LEARN_UNION are stated after Theorem 1 and in Definition 3.

Theorem 1: The algorithm LEARN_UNION of Fig. 6 exactly identifies any set $\mathcal{T}_{*} \in \mu O \mathcal{T F}_{\Lambda}$ in polynomial time using at most $m+1$ Equivalence queries and at most $2 m n^{2}$ Restricted Subset queries, where $m=\left|\mathcal{T}_{*}\right|, n$ is the maximum size of counterexamples and $|\Lambda|$ is infinite.

First, we consider the internal repeat-loop between lines 5 and 12 in the algorithm LEARN_UNION. For a linear term tree $t$, let $t^{\prime}$ be a linear term tree obtained from $t$ by replacing an edge $e$ of $t$ with a new variable. Then, $t^{\prime}$ is a generalized linear term tree of $t$ such that $t \leq t^{\prime}$. Let $t=\left(V_{t}, E_{t}, H_{t}\right) \in \mu O \mathcal{T T}_{\Lambda}$ and $e=(u, v) \in E_{t}$. We denote by $t /\{e\}$ the linear term tree obtained from $t$ by replacing the edge $e$ with a new variable $[u, v]$, that is, $t /\{e\}=$ $\left(V_{t}, E_{t}-\{e\}, H_{t} \cup\{[u, v]\}\right)$. For example, for a linear term

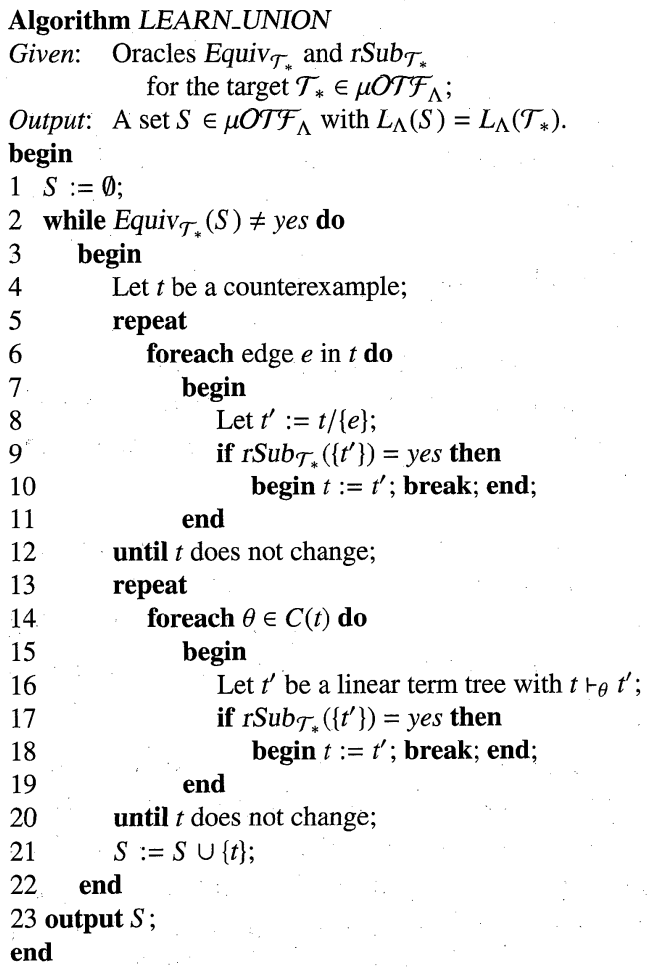

Fig. 6 Learning algorithm LEARN_UNION. We denote by Equiv $\mathcal{T}_{*}$ Equivalence query and by $r S u b_{\mathcal{T}_{*}}$ Restricted Equivalence query. 

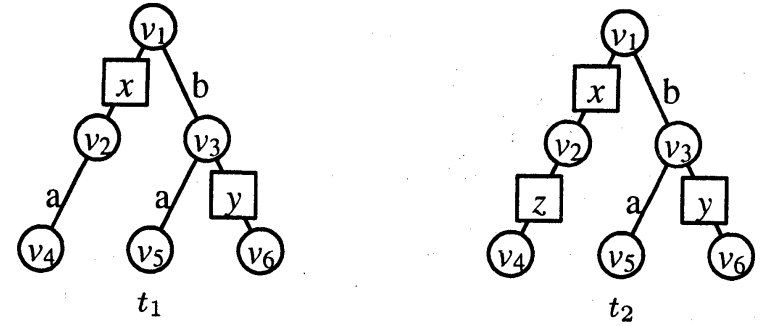

Fig. 7 Linear term trees $t_{1}$ and $t_{2}$ such that $t_{2} \equiv t_{1} /\left\{\left(v_{2}, v_{4}\right)\right\}$.

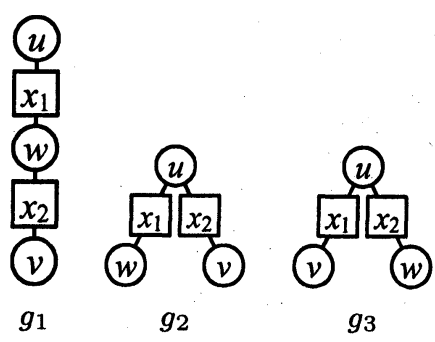

Fig. 8 Linear term trees $g_{1}, g_{2}, g_{3}$.

tree $t_{1}$ in Fig. 7, we give a linear term tree $t_{2}=t_{1} /\left\{\left(v_{2}, v_{4}\right)\right\}$ in Fig. 7 obtained from $t$ by replacing the edge $e=\left(v_{2}, v_{4}\right)$ with a new variable $\left[v_{2}, v_{4}\right]$. We can see that $t_{1} \leq t_{2}$. Therefore, we have the following lemma.

Lemma 3: Let $t=\left(V_{t}, E_{t}, H_{t}\right) \in \mu O \mathcal{T T}_{\Lambda}$ and $e \in E_{t}$. Then $t \leq t /\{e\}$.

For a linear term tree $t$ given after executing the internal repeat-loop between lines 5 and 12 in the algorithm $L E A R N_{-} U N I O N$, we can see that $L_{\Lambda}(t) \subseteq L_{\Lambda}\left(\mathcal{T}_{*}\right)$ and $L_{\Lambda}(t /\{e\}) \nsubseteq L_{\Lambda}\left(\mathcal{T}_{*}\right)$ for any $e \in E_{t}$. Then, we have the following lemma.

Lemma 4: Let $t=\left(V_{t}, E_{t}, H_{t}\right) \in \mu O \mathcal{T T}_{\Lambda}$ and $S \in \mu O \mathcal{T F}_{\Lambda}$. If $L_{\Lambda}(t) \subseteq L_{\Lambda}(S)$ and $L_{\Lambda}(t /\{e\}) \nsubseteq L_{\Lambda}(S)$ for any $e \in E_{t}$, then there exists a linear term tree $t^{\prime}=\left(V_{t^{\prime}}, E_{t^{\prime}}, H_{t^{\prime}}\right) \in S$ such that $t \leq t^{\prime}$ and $\left|E_{t}\right|=\left|E_{t^{\prime}}\right|$.

Proof. Since $L_{\Lambda}(t) \subseteq L_{\Lambda}(S)$, there exists a linear term tree $t^{\prime}=\left(V_{t^{\prime}}, E_{t^{\prime}}, H_{t^{\prime}}\right) \in S$ such that $t \leq t^{\prime}$ by Lemma 1 . Since $t \leq t^{\prime}$, we have $\left|E_{t^{\prime}}\right| \leq\left|E_{t}\right|$. We assume $\left|E_{t^{\prime}}\right|<\left|E_{t}\right|$. There exists an edge $e \in E_{t}$ such that $t /\{e\} \leq t^{\prime}$. This implies $L_{\Lambda}(t /\{e\}) \subseteq L_{\Lambda}(S)$. This is a contradiction. Therefore, we have $\left|E_{t}\right|=\left|E_{t^{\prime}}\right|$.

Second, we consider the internal repeat-loop between lines 13 and 20 in LEARN_UNION.

Definition 3: For linear term trees $t, t^{\prime}$ and a substitution $\theta=\{x:=[g,[u, v]]\}$, we write $t r_{\theta} t^{\prime}$ if $t^{\prime} \theta \equiv t$ and $g$ is one of the following linear term trees $g_{1}, g_{2}$ and $g_{3}$ (see Fig. 8).

1. $g_{1}=(\{u, v, w\}, \emptyset,\{[u, w],[w, v]\})$.

2. $g_{2}=(\{u, v, w\}, \emptyset,\{[u, w],[u, v]\})$ where $w<<_{u}^{g_{2}} v$.

3. $g_{3}=(\{u, v, w\}, \emptyset,\{[u, v],[u, w]\})$ where $v<_{u}^{g_{3}} w$.

If $\theta$ is clear from the context, we may write $r$ instead of $r_{\theta}$.

For linear term trees $t=\left(V_{t}, E_{t}, H_{t}\right)$ and $t^{\prime}=\left(V_{t^{\prime}}, E_{t^{\prime}}, H_{t^{\prime}}\right)$ in $\mu O \mathcal{T T}_{\Lambda}$, if $t \mathrm{r}_{\theta} t^{\prime}$, then there are three vertices $u_{1}, u_{2}, u_{3}$ such that $t^{\prime}$ can be transformed from $t$ by applying the following operations to $t$.

1. Contracting a subgraph consisting of two variables $\left[u_{1}, u_{2}\right],\left[u_{2}, u_{3}\right]$ in $t$ such that $u_{2}$ has exactly one child port in $t$ into the variable $\left[u_{1}, u_{3}\right]$, that is $V_{t^{\prime}}:=V_{t}-\left\{u_{2}\right\}$, $E_{t^{\prime}}:=E_{t}$ and $H_{t^{\prime}}:=H_{t} \cup\left\{\left[u_{1}, u_{3}\right]\right\}-\left\{\left[u_{1}, u_{2}\right],\left[u_{2}, u_{3}\right]\right\}$.

2. Removing a variable $\left[u_{1}, u_{2}\right]$ from $t$ if there exists a variable $\left[u_{1}, u_{2}\right]$ in $t$ such that $u_{2}$ is a leaf of $t$ and $u_{3}$ is the next sibling of $u_{2}$, that is $V_{t^{\prime}}:=V_{t}-\left\{u_{2}\right\}, E_{t^{\prime}}:=E_{t}$ and $H_{t^{\prime}}:=H_{t}-\left\{\left[u_{1}, u_{2}\right]\right\}$.

3. Removing a variable $\left[u_{1}, u_{3}\right]$ from $t$ if there exists a variable $\left[u_{1}, u_{3}\right]$ in $t$ such that $u_{3}$ is a leaf of $t$ and $u_{3}$ is the next sibling of $u_{2}$, that is $V_{t^{\prime}}:=V_{t}-\left\{u_{3}\right\}, E_{t^{\prime}}:=E_{t}$ and $H_{t^{\prime}}:=H_{t}-\left\{\left[u_{1}, u_{3}\right]\right\}$.

For example, in Fig. 9, $f_{1} \vdash_{\theta_{1}} f_{2}, f_{3} \vdash_{\theta_{2}} f_{4}$ and $f_{5} \vdash_{\theta_{3}} f_{6}$, where $\theta_{1}=\left\{x_{1}:=\left[g_{1},[u, v]\right]\right\}, \theta_{2}=\left\{x_{2}:=\left[g_{2},[u, v]\right]\right\}$, $\theta_{3}=\left\{x_{1}:=\left[g_{3},[u, v]\right]\right\}$ and $g_{1}, g_{2}, g_{3}$ are described in Fig. 8 . We can see that $f_{1} \leq f_{2}, f_{3} \leq f_{4}$ and $f_{5} \leq f_{6}$.

For a linear term tree $t=\left(V_{t}, E_{t}, H_{t}\right)$, we denote by $C(t)$ the set of all substitutions $\theta$ with $t r_{\theta} t^{\prime}$. Note that $|C(t)|<|t|$.

For linear term trees $t, t^{\prime}$, if $t r_{\theta} t^{\prime}$ then $t^{\prime}$ is a generalized linear term tree of $t$ such that $t \leq t^{\prime}$. Therefore we have the following lemmas.

Lemma 5: Let $t$ and $t^{\prime}$ be linear term trees in $\mu O \mathcal{T T}_{\Lambda}$. If $t \vdash t^{\prime}$, then $t \leq t^{\prime}$.

For a linear term tree $t$ given after executing the internal repeat-loop between lines 13 and 20 in the algorithm $L E A R N_{-} U N I O N$, we can see that $L_{\Lambda}\left(t^{\prime \prime}\right) \nsubseteq L_{\Lambda}\left(\mathcal{T}_{*}\right)$ for any $t^{\prime \prime} \in \mu O \mathcal{T T}_{\Lambda}$ with $t \vdash t^{\prime \prime}$.

Lemma 6: Let $t=\left(V_{t}, E_{t}, H_{t}\right)$ be a linear term tree in $\mu O \mathcal{T T}_{\Lambda}$ and $S$ a set in $\mu O \mathcal{T F}_{\Lambda}$ such that there exists a linear term tree $t^{\prime}=\left(V_{t^{\prime}}, E_{t^{\prime}}, H_{t^{\prime}}\right) \in S$ with $t \leq t^{\prime}$ and $\left|E_{t}\right|=\left|E_{t^{\prime}}\right|$. If $L_{\Lambda}\left(t^{\prime \prime}\right) \nsubseteq L_{\Lambda}(S)$ for any $t^{\prime \prime} \in \mu O \mathcal{T T}_{\Lambda}$ with $t \vdash t^{\prime \prime}$, then $t \equiv t^{\prime}$.

Proof. Since $t \leq t^{\prime}$, we have $|t| \geq\left|t^{\prime}\right|$. We assume $|t|>\left|t^{\prime}\right|$. Since $t \leq t^{\prime}$, there exists a substitution $\theta=$ $\left\{x_{1}:=\left[g_{1}, \sigma_{1}\right], \ldots, x_{n}:=\left[g_{n}, \sigma_{n}\right]\right\}$ such that $t \equiv t^{\prime} \theta$. Since $\left|E_{t}\right|=\left|E_{t^{\prime}}\right|$, we have $\left|H_{t}\right|>\left|H_{t^{\prime}}\right|$ by the assumption. We assume $\left|g_{i}\right|=2$ for any binding $x_{i}:=\left[g_{i}, \sigma_{i}\right]$ in $\theta(1 \leq i \leq n)$. Then we have $|t| \neq\left|t^{\prime} \theta\right|$. This contradicts with $t \equiv t^{\prime} \theta$. Next, we assume that there exists a binding $x_{i}:=\left[g_{i}, \sigma_{i}\right]$ in $\theta$ such that $E_{g_{i}} \neq \emptyset$, where $g_{i}=\left(V_{g_{i}}, E_{g_{i}}, H_{g_{i}}\right)(1 \leq i \leq n)$. Then, we have $\left|E_{t}\right|=\left|E_{t^{\prime}}\right| \neq\left|E_{t^{\prime} \theta}\right|$, where $t^{\prime} \theta=\left(V_{t^{\prime} \theta}, E_{t^{\prime} \theta}, H_{t^{\prime} \theta}\right)$. This contradicts with $t \equiv t^{\prime} \theta$. Therefore, there exists a binding $x_{\ell}:=\left[g_{\ell}, \sigma_{\ell}\right]$ in $\theta$ such that $\left|g_{\ell}\right| \geq 3$ and $E_{g_{\ell}}=\emptyset$, where $g_{\ell}=\left(V_{g_{\ell}}, E_{g_{\ell}}, H_{g_{\ell}}\right)(1 \leq \ell \leq n)$. We can construct a new substitution $\theta^{\prime}$ such that $t \equiv t^{\prime} \theta \vdash t^{\prime} \theta^{\prime}$ and $t^{\prime} \theta^{\prime} \in \mu O \mathcal{T T}_{\Lambda}$. By Lemma 5, we have $L_{\Lambda}(t) \subseteq L_{\Lambda}\left(t^{\prime} \theta^{\prime}\right) \subseteq L_{\Lambda}\left(t^{\prime}\right) \subseteq L_{\Lambda}(S)$. This is a contradiction. Thus, $|t|=\left|t^{\prime}\right|$. Since $\left|E_{t}\right|=\left|E_{t^{\prime}}\right|$ and $|t|=\left|t^{\prime}\right|$, we have $\left|H_{t}\right|=\left|H_{t^{\prime}}\right|$. Therefore, $t \equiv t^{\prime}$.

Let $S_{0}, S_{1}, \ldots, S_{i}, \ldots$ and $t_{1}, t_{2}, \ldots, t_{i}, \ldots(i \geq 0)$ be the sequence of hypotheses asked in the Equivalence queries by LEARN_UNION and the sequence of counterexamples 

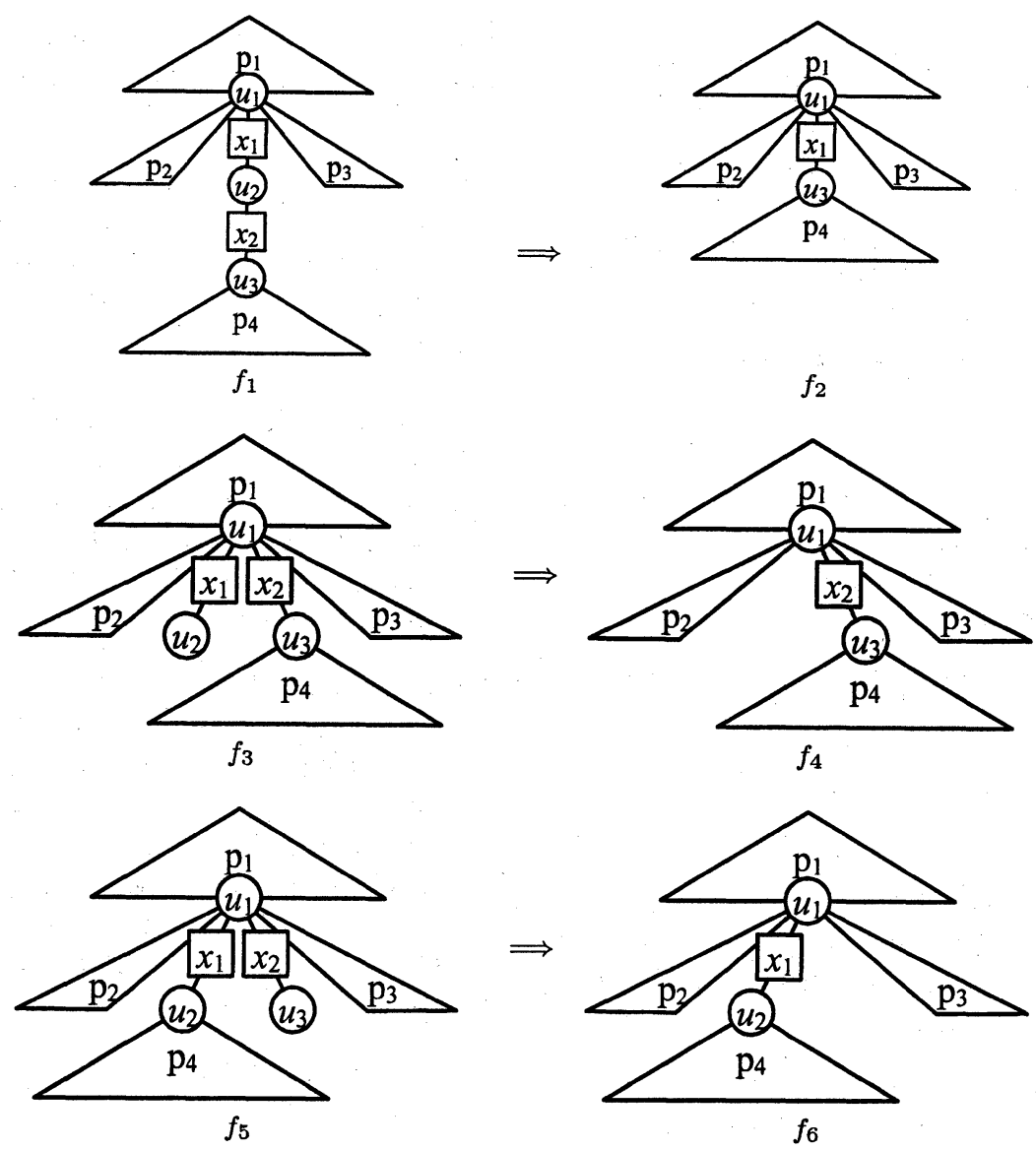

Fig. 9 Operations 1, 2 and 3 constructing linear term trees $f_{2}, f_{4}, f_{6}$ from linear term trees $f_{1}, f_{3}, f_{5}$, respectively.

returned by the queries respectively. Let $S_{0}$ be the initial hypothesis $\emptyset$. And we suppose that at each stage $i \geq 1$, LEARN_UNION makes an Equivalence query $\operatorname{Equiv}_{\mathcal{T}_{*}}\left(S_{i-1}\right)$, and receives a counterexample $t_{i}$ to the query.

Let $P, Q \in \mu O \mathcal{T F}_{\Lambda}$. If there exists a linear term tree $q \in Q$ such that $p \leq q$ for any $p \in P$, we write $P \sqsubseteq Q$. If $P \sqsubseteq Q$ and $P \nsupseteq Q$, then we write $P \sqsubset Q$.

Lemma 7: For each $i \geq 0, t_{i} \in L_{\Lambda}\left(\mathcal{T}_{*}\right)$ and $S_{i} \sqsubseteq \mathcal{T}_{*}$.

Proof. The proof is by the induction on the number of iterations $i \geq 0$ of the main loop. If $i=0$, then it is clear.

We assume inductively that the result holds for any number of iterations of the main loop less than $i$. By the inductive hypothesis, we have $S_{i-1} \sqsubseteq \mathcal{T}_{*}$. If $L_{\Lambda}\left(S_{i-1}\right) \neq$ $L_{\Lambda}\left(\mathcal{T}_{*}\right), t_{i}$ is obtained. Since $S_{i-1} \sqsubseteq \mathcal{T}_{*}$, we have $L_{\Lambda}\left(S_{i-1}\right) \subseteq$ $L_{\Lambda}\left(\mathcal{T}_{*}\right)$. Thus, $t_{i} \in L_{\Lambda}\left(\mathcal{T}_{*}\right)$.

Let $t_{i}^{\prime}=\left(V_{t_{i}^{\prime}}, E_{t_{i}^{\prime}}, S_{t_{i}^{\prime}}\right)$ be a linear term tree obtained by executing the first repeat-loop from $t_{i}$. By Lemma 1 and Lemma 4 , there exists a linear term tree $t_{*}^{\prime} \in \mathcal{T}_{*}$ such that $\left|E_{t_{i}^{\prime}}\right|=\left|E_{t_{*}^{\prime}}\right|$ and $t_{i}^{\prime} \leq t_{*}^{\prime}$. Let $t_{i}^{\prime \prime}$ be a linear term tree obtained by executing the second repeat-loop from $t_{i}^{\prime}$. By Lemma 1 and Lemma 6, there exists a linear term tree $t_{*}^{\prime \prime} \in \mathcal{T}_{*}$ such that $t_{i}^{\prime \prime} \equiv t_{*}^{\prime \prime}$. By the inductive hypothesis, we know $S_{i-1} \sqsubseteq$ $\mathcal{T}_{*}$. Therefore, we have $S_{i}=S_{i-1} \cup\left\{t_{i}^{\prime \prime}\right\} \sqsubseteq \mathcal{T}_{*}$.
Lemma 8: For each $i \geq 1, S_{i-1} \sqsubset S_{i}$.

Proof. For each $i \geq 1$, it is clear that $S_{i-1} \sqsubseteq S_{i}$. We assume that $S_{i-1} \sqsupseteq S_{i}$ for some $i \geq 1$. Let $t_{*}$ be a linear term tree which is added to $S_{i-1}$ at the $i$-th stage, that is, $S_{i}=S_{i-1} \cup$ $\left\{t_{*}\right\}$. Since $S_{i-1} \sqsupseteq S_{i}$, there exists a linear term tree $t_{*}^{\prime} \in S_{i-1}$ such that $t_{*} \equiv t_{*}^{\prime}$. By Lemma 3 and Lemma 5 , we have $t_{i} \leq t_{*} \equiv t_{*}^{\prime}$. This implies $t_{i} \in L_{\Lambda}\left(t_{*}\right)=L_{\Lambda}\left(t_{*}^{\prime}\right) \subseteq L_{\Lambda}\left(S_{i-1}\right)$. Since $t_{i}$ is an output counterexample by $\operatorname{Equiv}_{\mathcal{T}_{*}}\left(S_{i-1}\right)$, we have $t_{i} \notin L_{\Lambda}\left(S_{i-1}\right)$. This is a contradiction. lows.

By the above lemmas, we can prove Theorem 1 as fol-

Proof of Theorem 1. By the construction of the algorithm LEARN_UNION, Lemma 7 and Lemma 8, we know that the algorithm terminates and outputs $S$ such that $L_{\Lambda}(S)=$ $L_{\Lambda}\left(\mathcal{T}_{*}\right)$.

At the $i$-th stage of the while-loop, we receive a counterexample $t_{i}$. The loop of lines 6-11 uses at most $\left|t_{i}\right|$ Restricted Subset queries. Since the loop of lines 5-12 is repeated at most $\left|t_{i}\right|$ times, the loop uses at most $\left|t_{i}\right|^{2}$ Restricted Subset queries. We can show that the loop of lines 13-20 uses at most $\left|t_{i}\right|^{2}$ Restricted Subset queries in a similar way. Note that $\left|\mathcal{T}_{*}\right|=m$ and $n$ is the maximum size of counterexamples. By Lemma 7 and Lemma 8, since the while-loop is repeated at most $m$ times. Therefore, LEARN_UNION uses 
at most $2 m n^{2}$ Restricted Subset queries and at most $m+1$ Equivalence queries.

\section{Hardness Result on Learnability}

In this section, we show the insufficiency of learning of $\mu O \mathcal{T F} \mathcal{F}_{\Lambda}$ in exact learning model. We use the following lemma to show the insufficiency of learning of $\mu O \mathcal{T F} \mathcal{F}_{\Lambda}$.

Lemma 9: (László Lovász [9]) Let $U T_{n}$ be the number of all rooted unordered trees of size $n$ with no edge label. Then, $2^{n}<U T_{n}<4^{n}$, where $n \geq 6$.

We denote by $O T_{n}$ the set of all rooted ordered trees of size $n$ with no edge label. From the above lemma, we have $O T_{n}>2^{n}$, where $n \geq 6$. The following lemma is known to show the insufficiency of learning in exact learning model.

Lemma 10: (Angluin [4]) Suppose that a hypothesis space contains a class of distinct sets $L_{1}, \ldots, L_{N}$. If there exists a set $L_{\cap}$ in the hypothesis space such that for any pair of distinct indices $i$ and $j(1 \leq i, j \leq N), L_{\cap}=L_{i} \cap L_{j}$, then any algorithm that exactly identifies each of the hypotheses $L_{i}$ using Restricted Equivalence, Membership and Subset queries must make at least $N-1$ queries in the worst case.

By Lemma 9 and Lemma 10, we have Theorem 2.

Theorem 2: Any learning algorithm that exactly identifies all finite sets of linear term trees of size $n$ using Restricted Equivalence, Membership and Subset queries must make $\Omega\left(2^{n}\right)$ queries in the worst case, where $n \geq 6$ and $|\Lambda| \geq 1$.

Proof. We denote by $\mathcal{S}_{n}$ the class of all singleton sets of ground term trees of size $n$. The class $\mathcal{S}_{n}$ is a subclass of $\mu O \mathcal{T F} \mathcal{F}_{\Lambda}$ and for any $L$ and $L^{\prime}$ in $\mathcal{S}_{n}, L \cap L^{\prime}=\emptyset$. Since the empty set $L_{\Lambda}(\emptyset)=\emptyset$ is a hypothesis in $\mu O \mathcal{T F} \mathcal{F}_{\Lambda}$, by Lemmas 9 and 10, any learning algorithm that exactly identifies all finite sets of linear term trees of size $n$ using Restricted Equivalence, Membership and Subset queries must make $\Omega\left(2^{n}\right)$ queries in the worst case, even when $|\Lambda|=1$.

\section{Conclusion}

We have discussed the learnability of $\mu O \mathcal{T F} \mathcal{F}_{\Lambda}$ in the exact learning model. In Sect. 3, we have shown that any finite set $\mathcal{T}_{*}$ of linear term trees is exactly identifiable using at most $2 m n^{2}$ Restricted Subset queries and at most $m+1$ Equivalence queries, where $m=\left|\mathcal{T}_{*}\right|, n$ is the maximum size of counterexamples and $|\Lambda|$ is infinite.

Moreover, as a negative result we have shown that it is hard to exactly identify any set in $\mu O \mathcal{T F}_{\Lambda}$ efficiently using Restricted Equivalence, Membership and Subset queries. In the exact learning model using queries, other positive or negative learnability of $\mu O \mathcal{T F}_{\Lambda}$ is not known. As future work, we will investigate it.

We denote by $\mathcal{O T \mathcal { F } _ { \Lambda }}$ the set of all term trees with repeated variables. In [13], we showed the polynomial time learnability of $\mathcal{O T F}_{\Lambda}$ in the exact learning model by using Superset queries and Restricted Equivalence queries.
Table 1 Our results and future works.

\begin{tabular}{|c|c|c|}
\hline & Exact learning & $\begin{array}{l}\text { Inductive inference } \\
\text { from positive data }\end{array}$ \\
\hline$\mu O \mathcal{T T}_{\Lambda}$ & $\begin{array}{l}\text { Yes [12] } \\
|\Lambda| \geq 2\end{array}$ & $\begin{array}{c}\text { Yes [15] } \\
\text { polynomial time } \\
|\Lambda| \geq 1 \\
\end{array}$ \\
\hline$\left(\mu O \mathcal{T T}_{\Lambda}\right)^{2}$ & \multirow{2}{*}{$\begin{array}{l}\text { Yes [this work] } \\
\text { Equivalence \& } \\
\text { Restricted Subset } \\
|\Lambda| \text { is infinite }\end{array}$} & $\begin{array}{c}\text { Yes [8] } \\
\text { polynomial time } \\
|\Lambda| \text { is infinite }\end{array}$ \\
\hline$\mu O \mathcal{T F}_{\Lambda}$ & & Open \\
\hline $\mathcal{O T F}_{\Lambda}$ & $\begin{array}{c}\text { Yes [13] } \\
|\Lambda| \text { is infinite }\end{array}$ & Open \\
\hline
\end{tabular}

Suzuki et al. [15] showed the learnability of $\mu O \mathcal{T T}_{\Lambda}$ in the framework of polynomial time inductive inference from positive data [3]. Hirashima showed the learnability of the class of unions of two linear term tree languages, denoted by $\left(\mu O \mathcal{T T}_{\Lambda}\right)^{2}$, in the same framework [8]. For learning models other than the exact learning model and inductive inference from positive data, no results on the learnability of $\mu O \mathcal{T F}_{\Lambda}$ are known. Thus, we will study the learnabilities of $O \mathcal{T F}_{\Lambda}$ in the same framework. We summarize our results and future works in Table 1.

\section{References}

[1] T.R. Amoth, P. Cull, and P. Tadepalli, "Exact learning of tree patterns from queries and counterexamples," Proc. COLT-98, pp.175-186, ACM Press, 1998.

[2] T.R. Amoth, P. Cull, and P. Tadepalli, "On exact learning of unordered tree patterns," Mach. Learn., vol.44, pp.211-243, 2001.

[3] D. Angluin, "Finding pattern common to a set of strings,"J. Comput. Syst. Sci., vol.21, pp.46-62, 1980.

[4] D. Angluin, "Queries and concept learning," Mach. Learn., vol.2, pp.319-342, 1988.

[5] H. Arimura, H. Ishizaka, and T. Shinohara, "Learning unions of tree patterns using queries," Theor. Comput. Sci., vol.185, pp.4762, 1997.

[6] H. Arimura, H. Sakamoto, and S. Arikawa, "Efficient learning of semi-structured data from queries," Proc. ALT-2001, LNAI 2225, pp.315-331, Springer-Verlag, 2001.

[7] H. Arimura, T. Shinohara, and S. Otsuki, "Polynomial time algorithm for finding finite unions of tree pattern languages," Proc. NIL91, LNAI 659, pp.118-131, Springer-Verlag, 1993.

[8] H. Hirashima, Y. Suzuki, S. Matsumoto, T. Uchida, and Y. Nakamura, "Polynomial time inductive inference of unions of two term tree languages," Proc. ILP-2006, (Short Papers) The University of Corunna, UDC Press service, 2006.

[9] L. Lovász, Combinatorial Problems and Exercises, chapter Two classical enumeration problems in graph theory, North-Holland Publishing Company, 1979.

[10] S. Matsumoto and A. Shinohara, "Learning pattern languages using queries," Proc. EuroCOLT-97, LNAI 1208, pp.185-197, SpringerVerlag, 1997.

[11] S. Matsumoto, A. Shinohara, H. Arimura, and T. Shinohara, "Learning subsequence languages," in Information Modelling and Knowledge Bases VIII, pp.335-344, IOS Press, 1997.

[12] S. Matsumoto, T. Shoudai, T. Miyahara, and T. Uchida, "Learning unions of term tree languages using queries," Proc. LA Summer Symposium, pp.21-1-21-10, July 2002. 
[13] S. Matsumoto, Y. Suzuki, T. Shoudai, T. Miyahara, and T. Uchida, "Learning of finite unions of tree patterns with repeated internal structured variables from queries," Proc. ALT-2003, LNAI 2842, pp.144-158, Springer-Verlag, 2003.

[14] T. Miyahara, Y. Suzuki, T. Shoudai, T. Uchida, K. Takahashi, and H. Ueda, "Discovery of frequent tag tree patterns in semistructured web documents," Proc. PAKDD-2002, LNAI 2336, pp.341-355, Springer-Verlag, 2002.

[15] Y. Suzuki, R. Akanuma, T. Shoudai, T. Miyahara, and T. Uchida, "Polynomial time inductive inference of ordered tree patterns with internal structured variables from positive data," Proc. COLT-2002, LNAI 2375, pp.169-184, Springer-Verlag, 2002.

[16] Y. Suzuki, Y. Inomae, T. Shoudai, T. Miyahara, and T. Uchida, "A polynomial time matching algorithm of structured ordered tree patterns for data mining from semistructured data," Proc. ILP-02, LNAI 2583, pp.270-284, Springer-Verlag, 2002.

[17] Y. Suzuki, T. Shoudai, S. Matsumoto, and T. Miyahara, "Polynomial time inductive inference of ordered tree languages with height-constrained variables from positive data," Proc. PRICAI2004, LNAI 3157, pp.211-220, Springer-Verlag, 2004.

[18] Y. Suzuki, T. Shoudai, T. Uchida, and T. Miyahara, "Ordered term tree languages which are polynomial time inductively inferable from positive data," Theor. Comput. Sci., vol.350, pp.63-90, 2006.

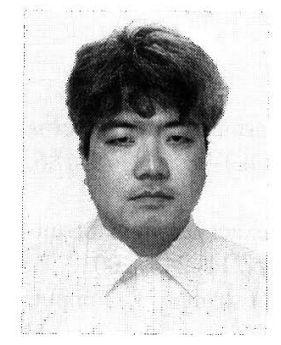

Satoshi Matsumoto is an associate professor of Department of Mathematical Sciences, Tokai University, Kanagawa, Japan. He received the B.S. degree in Mathematics, the M.S. and Dr. Sci. degrees in Information Systems all from Kyushu University, Fukuoka, Japan in 1993, 1995 and 1998, respectively. His research interests include algorithmic learning theory.

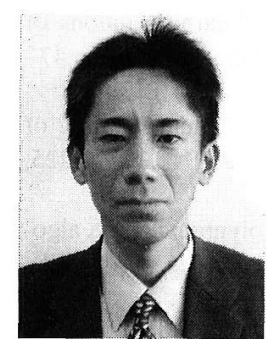

Takayoshi Shoudai received the B.S. in 1986, the M.S. degrees in 1988 in Mathematics and the Dr. Sci. in 1993 in Information Science all from Kyushu University. Presently, he is an associate professor of Department of Informatics, Kyushu University. His present interests include algorithmic graph theory, computational complexity, computational learning theory. He is a member of IPSJ and ACM.

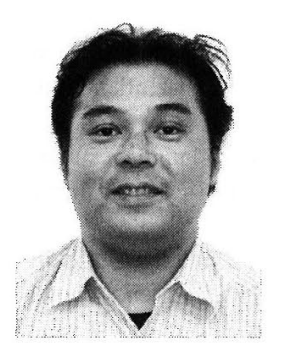

Tomoyuki Uchida received the B.S. degree in Mathematics, the M.S. and Dr. Sci. degrees in Information Systems all from Kyushu University, in 1989, 1991 and 1994, respectively. Currently, he is an associate professor of Graduate School of Information Sciences, Hiroshima City University. His research interests include data mining from semistructured data, algorithmic graph theory and algorithmic learning theory. He is a member of ACM.

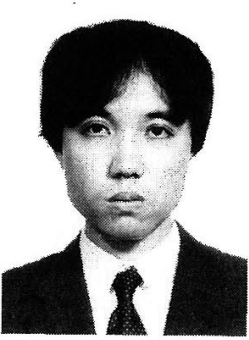

Tetsuhiro Miyahara

is an associate professor of Graduate School of Information Sciences, Hiroshima City University, Hiroshima, Japan. He received the B.S. degree in Mathematics, the M.S. and Dr. Sci. degrees in Information Systems all from Kyushu University, Fukuoka, Japan in 1984, 1986 and 1996, respectively. His research interests include algorithmic learning theory, knowledge discovery, inductive logic programming and machine learning.

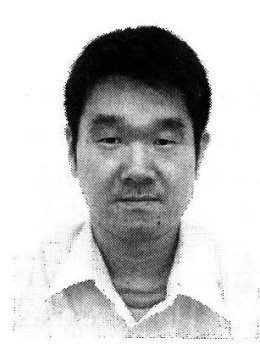

Yusuke Suzuki received the B.S. degree in Physics, the M.S. and Dr. Sci. degrees in Informatics all from Kyushu University, in 2000, 2002 and 2007 , respectively. He is currently a research associate of Graduate School of Information Sciences, Hiroshima City University, Hiroshima, Japan. His research interests include Machine Learning and Data Mining. 